Service social

\title{
Qui sont et que font les travailleurs sociaux en réadaptation en déficience physique?
}

\section{Josianne Crête}

Volume 62, numéro 2, 2016

URI : https://id.erudit.org/iderudit/1038578ar

DOI : https://doi.org/10.7202/1038578ar

Aller au sommaire du numéro

\section{Éditeur(s)}

École de travail social et de criminologie de l’Université Laval

ISSN

1708-1734 (numérique)

Découvrir la revue

Citer cet article

Crête, J. (2016). Qui sont et que font les travailleurs sociaux en réadaptation en déficience physique? Service social, 62(2), 80-93.

https://doi.org/10.7202/1038578ar
Résumé de l'article

Le travail social se centrant sur le lien entre l'individu et son environnement, il apparaît pouvoir contribuer à comprendre le handicap en augmentant l'attention donnée aux facteurs environnementaux, jusqu'à maintenant dévalorisés au profit des facteurs individuels. Toutefois, puisque tout projet disciplinaire s'actualise dans un dialogue avec son contexte, il devient intéressant d'explorer la réelle expression de l'identité professionnelle des travailleurs sociaux en réadaptation en déficience physique, ce qu'un projet doctoral a fait grâce à la méthodologie de la théorisation enracinée. Les résultats démontrent que, à partir d'une conception du travail social en partie cohérente avec l'approche écologique, le contexte teinte la façon dont les participantes prennent en considération les facteurs environnementaux dans leurs rôles et tâches très variés. Ces résultats sont mis en dialogue avec les écrits scientifiques portant sur le sujet. 


\title{
Qui sont et que font les travailleurs sociaux en réadaptation en déficience physique?
}

\author{
CRÊTE, Josianne \\ Doctorante en service social \\ Université de Montréal
}

\begin{abstract}
RÉSUMÉ
Le travail social se centrant sur le lien entre l'individu et son environnement, il apparaît pouvoir contribuer à comprendre le handicap en augmentant l'attention donnée aux facteurs environnementaux, jusqu'à maintenant dévalorisés au profit des facteurs individuels. Toutefois, puisque tout projet disciplinaire s'actualise dans un dialogue avec son contexte, il devient intéressant d'explorer la réelle expression de l'identité professionnelle des travailleurs sociaux en réadaptation en déficience physique, ce qu'un projet doctoral a fait grâce à la méthodologie de la théorisation enracinée. Les résultats démontrent que, à partir d'une conception du travail social en partie cohérente avec l'approche écologique, le contexte teinte la façon dont les participantes prennent en considération les facteurs environnementaux dans leurs rôles et tâches très variés. Ces résultats sont mis en dialogue avec les écrits scientifiques portant sur le sujet.
\end{abstract}

Mots-clés : déficience physique, travail social, identité professionnelle, rôles professionnels, méthodologie de la théorisation enracinée

\begin{abstract}
Social work being a profession that concentrates on the relationship between individuals and their environment, it seems to be in a good position to help understand handicap by increasing the importance given to environmental factors, that long have been put aside in favor of individual factors. Although, since all disciplinary projects are actualized in a dialogue with their context, it becomes interesting to explore the real expression of the professional identity of social workers employed in rehabilitation settings for people who have physical disabilities, which has been done through a doctoral research. Results demonstrate that, starting with a conception of social work that is partly consistent with the ecological approach, the context influences the way participants take environmental factors into consideration in their diverse roles and tasks. Before bringing the article to a close, these results are discussed in comparison with scientific knowledge on the topic.
\end{abstract}

Key words: physical disabilities, social work, professional identity, professional roles, grounded theory 
Selon l'Ordre professionnel des travailleurs sociaux et thérapeutes conjugaux et familiaux du Québec (OTSTCFQ, 2012), la pratique du travail social est « la recherche de rapports satisfaisants entre les personnes et leurs contextes de vie, favorisant ainsi le bien-être, la participation sociale et citoyenne ainsi que le développement social » (p. 9). C'est donc par l'importance accordée aux liens entre l'individu et son environnement que cette profession se caractérise (Blewett, Lewis et Tunstill, 2007). Cela transparaît jusque dans les valeurs sur lesquelles repose le travail social, que Bouquet (2003) sépare en deux socles, les valeurs humanistes d'un côté, ayant l'humain comme préoccupation centrale, et les valeurs républicaines et démocratiques de l'autre, qui placent l'humain dans un contexte social et politique respectueux de ses droits.

Ce projet disciplinaire est en cohérence avec le mandat des établissements de réadaptation en déficience physique. En fait, ces établissements offrent des "services de réadaptation spécialisés, de soutien à l'intégration et des services de maintien dans le milieu de vie » (INESSS, 2012 b, p. 6). Ce sont des services qui visent à permettre à la personne admise en réadaptation interne de retourner habiter son milieu de vie, ou de s'en trouver un nouveau, adapté à sa situation, et à la personne inscrite en réadaptation externe de poursuivre à domicile sa récupération fonctionnelle (INESSS, 2012a). Cela passe par toute une gamme de services, allant des services de promotion et de prévention visant à diminuer les facteurs de risque, à des services de réadaptation ayant comme objectif de développer les capacités de la personne, en passant par « l'attribution d'aides techniques pour compenser les incapacités et pour réduire les obstacles environnementaux » (ASSSGIM, 2006, p. 4).

Cette idée d'obstacles environnementaux en lien avec la notion de handicap continue d'être débattue. Au Québec, Fougeyrollas et son équipe (1998) ont développé un cadre d'analyse rejetant la vision mécaniste et linéaire du modèle biomédical, individualisant, pour faire place à une vision holistique de la santé. II s'agit du Processus de production du handicap (PPH). Dans une logique écologique, ce modèle propose de situer le handicap non pas chez la personne ayant une déficience physique, mais plutôt dans la rencontre entre ses facteurs individuels (déficience physique incluse), ses facteurs environnementaux (facilitateurs et obstacles) et ses habitudes de vie. De cette façon, on ne parle plus de «personnes handicapées », mais plutôt de «personnes en situation de handicap ». Étudiant les retombées de ce modèle, Fougeyrollas (2010) se réjouit que le PPH ait gagné en popularité au Québec, tout en déplorant que cette popularité soit restée à un niveau plutôt théorique, n'ayant que peu d'effets dans la pratique des intervenants. Cela l'amène à conclure que «le processus de réadaptation semble caractérisé par une surestimation de l'influence des facteurs personnels » (p. 263) au détriment de la prise en compte des facteurs environnementaux dans l'intervention.

Étant donné que le travail social porte aussi cette vision écologique et se caractérise par le pont qu'il fait entre l'individu et son environnement, il pourrait être vu comme une discipline de choix pour se rapprocher de l'objectif du PPH en augmentant l'attention mise sur les facteurs environnementaux, sans pour autant mettre de côté les facteurs personnels. Par contre, il est bien connu que la façon dont s'actualise le projet disciplinaire dans un contexte de travail est influencée par les caractéristiques de ce contexte (Chouinard et Couturier 2006). C'est ce qu'affirme Payne (2004) en soulignant que l'identité «créée en négociant qui fait quoi dans les milieux de travail affecte les rôles et identités professionnels » (p. 6, traduction libre). Dans ce 
sens, il est intéressant de savoir que le rôle des travailleurs sociaux exerçant en réadaptation en déficience physique est considéré comme un rôle supplétif au « véritable » travail de réadaptation qui se fait dans ces établissements (Prud'homme, 2011, p. 58).

Ainsi, bien que portant une vision disciplinaire qui permettrait une plus grande prise en considération des facteurs environnementaux, les travailleurs sociaux œuvrant auprès de personnes ayant une déficience physique semblent relégués à un rôle de deuxième plan. Mais est-ce vraiment le cas ? Ce questionnement est à la base d'un projet de recherche doctoral s'intéressant à la façon dont s'exprime l'identité professionnelle des travailleurs sociaux qui œuvrent en réadaptation en déficience physique. En s'intéressant à ce sujet, l'identité professionnelle de ces intervenants est étudiée, mais aussi les rôles et tâches par lesquels cette identité s'exprime dans le quotidien, ainsi que les éléments du contexte qui influencent son expression concrète. Cela permet d'explorer en quoi leur rôle est supplétif ou non.

Dans le présent article, après avoir détaillé le cadre théorique et méthodologique guidant le projet, nous présentons les conceptions du travail social qu'ont les participantes ${ }^{1}$, puis la façon dont certaines caractéristiques du contexte influencent la mise en œuvre de ces conceptions. Ensuite, nous présentons les rôles et tâches que les participantes assument dans leur travail quotidien. Avant de conclure, ces résultats sont mis en dialogue avec les données scientifiques, entre autres afin de voir en quoi les travailleuses sociales jouent un rôle supplétif ou non, et de second ordre ou non.

\section{Identité professionnelle et travail social}

Pour arriver à notre objectif, nous avons étudié l'identité professionnelle qui, à sa plus simple expression, est comprise comme ce que le « je » s'approprie du « nous » auquel il appartient, tout en se distinguant du « eux » qu'il rejette (Legault, 2008). De façon plus complète, il s'agirait du « résultat à la fois stable et provisoire, individuel et collectif, subjectif et objectif, biographique et structurel, des divers processus de socialisation qui, conjointement, construisent les individus et définissent les institutions »(Dubar, 2000, p. 109). L'identité professionnelle se construit donc par la résolution de ces tensions, en réinterprétant les conditions objectives de l'identité du groupe professionnel auquel l'individu se rattache, ici le travail social (Granja, 2008). Concrètement, cela s'exprime à travers les rôles et les tâches que les professionnels assument dans leur équipe, ce qui varie selon les caractéristiques du contexte organisationnel (Chouinard et Couturier 2006), puisque les identités diffèrent selon les résultats de la négociation visant à savoir qui fera quoi dans ce contexte (Payne, 2004).

En travail social, diverses identités se côtoient. Strauss (1992) utilise le terme « segment professionnel » pour décrire l'hétérogénéité de l'identité de différentes professions, ce qui s'applique bien au travail social. Ainsi, il existe plusieurs visions de cette profession. À travers cette diversité, le choix est ici fait de rattacher notre analyse à l'approche écologique (Bronfenbrenner, 1994), puisqu'il s'agit à la fois d'une approche courante en travail social (PullenSansfaçon et Cowden, 2012) et celle qui est à la base du PPH (Fougeyrollas, 2010). C'est ce qui

\footnotetext{
${ }^{1}$ Le féminin est utilisé lorsqu'il est question des participantes au projet ici détaillé puisque l'échantillon est composé presque exclusivement de femmes.
} 
rend le travail social si cohérent avec le cadre d'analyse préconisé en réadaptation en déficience physique. Dans ce sens, les travailleurs sociaux porteraient une vision situant l'individu au centre des divers systèmes qui l'entourent, où tous ces systèmes - incluant l'individu, l'ontosystème sont en interaction et s'interinfluencent (Bouchard, 1987).

\section{Méthodologie}

Le projet dont il est ici question est basé sur la méthodologie de la théorisation enracinée constructiviste (Charmaz, 2000), qui représente à la fois un cadre méthodologique et une posture épistémologique. C'est donc dans une logique constructiviste, inductive et itérative que le projet est réalisé. La collecte de données s'est faite grâce à 20 entrevues individuelles en profondeur, qui ont eu lieu entre novembre 2014 et janvier 2015, en personne lorsque possible, et par média technologique lorsque la distance l'exigeait. La modalité choisie présente l'avantage de laisser place à la narration de soi et à la réflexivité, concepts centraux dans la construction identitaire (Ruohotie-Lyhty, 2013). De plus, son aspect exploratoire se conjugue bien à la logique inductive privilégiée (Charmaz, 2000). Lors des entrevues, différents thèmes ciblés ont été abordés, tels que le cheminement professionnel et académique des participantes, les caractéristiques de leur travail (contexte, clientèle, rôles, équipe, etc.), ainsi que leur identité professionnelle (leur définition de ce concept, de leur identité et de celle du travail social, etc.). De plus, dans une logique inductive, de la place a été laissée à l'émergence de nouveaux thèmes qui, pour les participantes, étaient en lien avec le sujet abordé, mais auquel nous n'avions pas préalablement pensé.

Pour avoir accès à l'expression de l'identité professionnelle des travailleuses sociales exerçant en réadaptation en déficience physique, nous avons décidé de cibler des personnes membres de I'OTSTCFQ qui, au moment de leur entrevue, travaillaient dans un établissement membre de l'AÉRDPQ ${ }^{2}$ depuis au moins un an. Le recrutement s'est donc fait par l'envoi d'un courriel aux membres de l'OTSTCFQ qui s'auto-déclarent comme travaillant auprès d'une clientèle ayant une déficience physique. Parmi ces destinataires, 20 ont répondu à l'annonce et ont été rencontrés, soit 19 femmes et un homme ${ }^{3}$. Celles-ci ont entre 1 an et 19 ans d'expérience comme travailleuse sociale en réadaptation en déficience physique, et sont réparties dans dix établissements différents parmi les 21 établissements qui étaient membres de l'AÉRDPQ. Notons que, même si plusieurs régions du Québec sont représentées dans l'échantillon, plus de la moitié des participantes (onze) proviennent de l'île de Montréal.

\section{Analyse des données}

Les entrevues réalisées ont été transcrites puis analysées à l'aide du logiciel NVivo 10. En cohérence avec la logique itérative proposée par la méthodologie de la théorisation enracinée, il a été décidé de débuter par une analyse partielle des données afin de construire une grille de

\footnotetext{
${ }^{2}$ Notons que l'Association des établissements de réadaptation en déficience physique du Québec (AÉRDPQ) a été dissoute en avril 2015, suite aux changements structurels majeurs découlant de l'adoption de la Loi modifiant l'organisation et la gouvernance du réseau de la santé et des services sociaux notamment par l'abolition des agences régionales (L-10).

3 Pour des raisons de confidentialité, les données provenant de cet homme ont été féminisées, et un pseudonyme féminin lui a été donné.
} 
codage qui a ensuite été révisée selon l'analyse des entrevues restantes. Quatorze entrevues ont ainsi d'abord été analysées à l'aide de stratégies caractéristiques de la méthodologie de la théorisation enracinée. Premièrement avec le codage ouvert, qui se fait en restant fidèle aux données empiriques. Ces codes sont ensuite comparés entre eux et avec les données empiriques et scientifiques grâce à la technique de la comparaison constante, permettant de les regrouper en catégories représentant un niveau d'abstraction plus élevé, soit le codage axial (Guillemette et Lapointe, 2012). Les six dernières entrevues ont ensuite été analysées à partir des codes ainsi identifiés, à l'aide cette fois de la comparaison constante et du codage axial. La rédaction de mémos analytiques et méthodologiques a facilité le processus d'analyse (Guillemette et Lapointe, 2012).

L'analyse s'est appuyée sur des concepts sensibilisateurs et notre sensibilité théorique, en mettant en rapport les données empiriques, les données scientifiques et notre expérience personnelle et professionnelle (Duchesne et Savoie-Zajc, 2005). Cela a permis l'émergence d'éléments théoriques enracinés dans les données recueillies. II faut ici souligner que notre posture constructiviste a un impact sur la façon dont les données sont analysées et sur la façon dont la théorisation se construit. En fait, "la méthodologie de la théorisation enracinée constructiviste distingue le réel du vrai. L'approche constructiviste ne recherche pas la vérité unique, universelle et intemporelle. Toutefois, elle demeure réaliste, car elle appréhende les réalités humaines et présume de l'existence d'un monde réel » (Charmaz, 2000, p. 523, traduction libre). Ainsi, la réalité devient une coconstruction entre nous et les participantes, où les données colligées sont marquées par la relation qui s'établit entre ces personnes, et où l'analyse de ces données reflète la lecture que nous en avons, selon notre sensibilité (Charmaz, 2000). Les résultats qui suivent représentent donc notre façon de raconter l'histoire que les participantes ont élaborée avec nous pendant les entrevues, dans une co-construction de leur réalité.

\section{Les travailleuses sociales se racontent}

Les entrevues réalisées avec les participantes sont riches en informations au sujet de la façon dont s'exprime leur identité professionnelle dans le contexte de la réadaptation en déficience physique. À cet effet, dans les pages qui suivent, nous détaillerons d'abord leurs conceptions de ce qu'est le travail social, en soulignant en quoi ces conceptions convergent ou divergent de celle proposée par l'approche écologique (Bronfenbrenner, 1994). Par la suite, nous exposerons quelques éléments du contexte ayant un impact sur la façon dont s'actualisent ces conceptions dans leur travail en réadaptation. Pour terminer, nous présenterons les rôles et les tâches par lesquels ces conceptions se concrétisent.

\section{Le travail social, c'est s'intéresser au lien entre l'individu et son environnement}

Moi, je trouve que notre identité, c'est vraiment la personne en lien avec son environnement. C'est ça que je trouve qui est le spécifique. En même temps, je me dis qu'il y a peut-être d'autres professions pour qui c'est un peu ça. [...] C'est que nos clients vivent des choses au quotidien, 
pas juste en lien avec leur déficience, mais aussi avec ce qu'ils ont à vivre dans leur environnement finalement. (Rose ${ }^{4}$ )

Les participantes mettent l'accent sur le lien entre la personne et son environnement, ce qui les amène à affirmer qu'elles travaillent « avec la globalité de la personne » (Chloé), par exemple grâce à «l'approche systémique » (Clara). Elles en viennent alors à se définir comme des généralistes, ce qui convient à certaines comme Ophélie, mais entraîne certaines difficultés, particulièrement quand vient le temps de se mettre en mots. C'est par exemple ce qu'explique Rose en soulignant que, en « travail social on est tellement un peu partout, à plein de niveaux, à faire des tâches tellement différentes d'un bord et de l'autre que, des fois, j'ai de la misère à dire ce que c'est une travailleuse sociale ». En fait, puisqu'il s'agit d'une discipline si « large », s'ensuit une « situation où on se demande à quoi les travailleurs sociaux servent » (Romane). Ainsi, pour les participantes, le propre de leur discipline est de se centrer sur le lien entre la personne et son environnement, grâce à une vision globale, les positionnant dans un rôle de généraliste, où mettre en mots qui elles sont et ce qu'elles font est un défi découlant parfois sur un questionnement au sujet de leur utilité.

Toutefois, bien qu'elles définissent peu ce qu'elles entendent par « environnement ", à travers leur discours, trois entités ressortent comme en faisant partie. II s'agit d'abord des proches, ce qui se résume pour plusieurs à la famille (par exemple, Camille et Zoé), et parfois même à un seul membre de la famille, comme pour Simone, qui affirme « on a bien plus souvent des mamans dans nos bureaux». Ensuite, elles parlent des organismes de la communauté, référant habituellement aux partenaires avec qui elles font affaire pour répondre aux besoins de la personne. «Les ressources [...] c'est comme aide sociale, loisirs, ça peut être l'agent payeur, ça peut être $\mathrm{HLM}^{5}$. II y a plein, plein, plein de partenaires avec qui on est appelées à travailler pour la clientèle» (Romane). Finalement, il s'agit de la réadaptation elle-même, comme environnement temporaire et provisoire de la personne, ce qui est sous-entendu dans leur discours par le nombre de rôles qu'elles jouent auprès de leurs collègues. Nous voyons donc que l'environnement sur lequel elles ciblent leurs interventions exclut certaines sphères, comme l'environnement physique, le contexte sociopolitique et le contexte socioculturel. Bien que cette absence dans leur discours ne veuille pas dire qu'elles n'en tiennent pas compte dans leurs interventions, cela vient ébranler la conception écologique que les travailleuses sociales sont centrées sur la personne dans la globalité de son environnement.

Certaines ébranlent encore cette idée par l'importance qu'elles accordent au «clinique », et par ce que ce terme veut dire pour elles. Par exemple, pour Charlotte, le «clinique » est ce qui distingue la travailleuse sociale des autres membres de l'équipe, surtout en l'absence de psychologue. Pour elle, le " clinique » est une façon de parler de relation d'aide, en se laissant guider par des concepts psychologiques, souvent reliés à la psychothérapie, "par exemple, le transfert, le contre-transfert », et les personnalités difficiles pour l'équipe « surtout si le patient ne veut pas rencontrer le psychologue de la place ». Cela laisse entendre qu'elle centre sa définition identitaire sur un individu, en psychologisant son vécu, l'éloignant encore de cette idée que les travailleuses sociales mettent l'accent sur le lien entre l'individu et son environnement.

\footnotetext{
${ }^{4}$ Tous les noms utilisés pour désigner les participantes sont des pseudonymes.

${ }^{5}$ Habitations à loyer modique
} 
On voit donc une certaine cohérence entre le discours des participantes et le discours de l'approche écologique en ce qui a trait à l'importance du lien entre l'individu et son environnement. Mais aussi quelques divergences. D'abord en raison de la définition qu'elles donnent au concept « d'environnement » et les sphères environnementales qu'elles incluent dans leur travail. Ensuite, en lien avec l'importance qu'elles accordent parfois aux théories psychologiques et psychothérapeutiques. Elles expliquent d'ailleurs que des caractéristiques du milieu justifient ces divergences. II apparaît donc pertinent de mieux comprendre quelles sont ces caractéristiques, et comment elles influent sur la façon dont les participantes conçoivent leur profession.

\section{Une construction identitaire teintée par le contexte de travail}

Les participantes expliquent que la mise en action de leurs conceptions du travail social est limitée par différents aspects de leur contexte de travail. Par exemple, pour expliquer qu'elles ne s'attardent qu'à certaines sphères de l'environnement de la clientèle, elles soulignent l'importance de « délimiter chacun qui fait quoi [... pour ne] pas se dédoubler » (Emma). Donc si un collègue d'une autre discipline travaille sur une partie de l'environnement, elles ne s'y attarderont pas. De la même façon, pour expliquer que leurs interventions auprès de la famille se limitent à l'intervention auprès d'un seul membre de la famille, certaines parlent des disponibilités des autres personnes autour de l'usager. Par exemple, Simone qui parle d'une famille qu'elle suit où elle ne rencontre que la mère puisque le père travaille sur la route et est régulièrement à l'extérieur de la ville. II paraît donc évident que, en plus d'une tendance à la psychologisation présente chez certaines, différents éléments de leur contexte expliquent que leurs conceptions du travail social soient transformées vers quelque chose de plus individualisant. À ce sujet, deux éléments méritent particulièrement notre attention, soit le mandat de l'établissement et le travail en équipe.

En ce qui concerne le mandat de l'établissement, bien que l'ensemble des intervenants travaille dans le même sens, chaque discipline s'y prend d'une façon différente. De façon générale, le mandat de réadaptation cible les personnes ayant la déficience physique et vise à « les rendre le plus fonctionnelles possible » (Clara) et à « améliorer leur qualité de vie » (Romane). Ce mandat est ce qui guide l'intervention des participantes. « Moi, j'essaye vraiment de rester par rapport à mon mandat en réadaptation » (Anaïs). Une des caractéristiques de ce mandat est qu'il cible une seule personne, et reste flou quant à la place accordée aux proches et à la famille. Sachant que, en « travail social, on travaille avec les familles » (Aurélie), ce flou devient central dans la relation qu'ont les participantes avec leur contexte de travail. En fait, certaines participantes considèrent que leurs clients sont à la fois l'usager et ses proches, alors que d'autres positionnent les proches plutôt dans un rôle secondaire.

Ça, c'est vraiment le droit de la personne, pas le besoin de sa fille ou de son parent, mais le besoin de mon client et ses droits à lui. Je comprends que la famille est épuisée. On va essayer de trouver des moyens, mais pas au détriment des décisions de la personne. Mais je sais que, pour d'autres, l'impact sur la famille a beaucoup de place dans leur façon d'intervenir. (Chloé)

On voit donc que le mandat joue un rôle dans la façon dont les participantes positionnent les proches des usagers. Cela teinte la façon dont leurs conceptions du travail social sont 
actualisées, prenant parfois une couleur plutôt familiale, et d'autres fois une couleur plutôt individualisante.

À travers ce mandat partagé, comme le rappelle Camille, dans l'équipe, « on a chacun nos tâches ». Chacun a un rôle qui lui est propre, bien que parfois partagé avec d'autres membres de l'équipe, ce que nous appelons les zones de partage. Bien que nous ayons peu exploré les rôles que jouent les autres membres de l'équipe, ce qui ressort de l'analyse des données est que les travailleuses sociales ne sont pas les seules à intervenir auprès des familles. Par exemple, travailler la communication dans la famille est une zone partagée entre Romane et l'orthophoniste de son équipe. Mia a une zone de partage avec les éducateurs spécialisés «qui travaillent à la maison sur l'intégration sociale et la reprise d'habitudes de vie et de routine, ils vont être en contact avec ma famille ». Elles sont alors appelées à trouver " une façon de travailler » (Zoé) avec les membres de l'équipe, pour départager les tâches qu'elles voient comme les leurs, démontrant une adaptabilité à leur contexte de travail dans ce partage de tâches. Toutefois, il semble qu'elles ne veulent pas se laisser définir par ce contexte, affirmant qu'il est « important de se positionner. Ça fait partie peut-être de nos lacunes [...] on attend les commandes des autres » (Clara). Les participantes semblent donc tiraillées entre se définir à travers leur contexte et imposer leur définition d'elles-mêmes.

\section{Faire de tout, même des miracles, pour faciliter la réadaptation}

Comme il vient d'être exposé, les participantes voient leurs conceptions du travail social influencées par le mandat qui leur est confié en réadaptation, ainsi que par les réalités du travail en équipe. De cette mise en rapport entre, d'un côté, leurs conceptions de leur discipline d'attache et, de l'autre, le contexte dans lequel ces conceptions s'actualisent, ressort une diversité de tâches et de rôles qu'elles assument. En fait, leurs tâches et rôles sont si variés que certaines en viennent à dire que les travailleuses sociales font de tout et de rien. Par contre, lorsque questionnée à cet effet, Chloé souligne que c'est plutôt le contraire, puisque faire de tout, «ce n'est pas rien ». La conception du travailleur social mettant l'accent sur le lien entre la personne et son environnement se traduit donc par un rôle que plusieurs qualifient de " généraliste » (par exemple, Ophélie et Anaïs), où elles sont appelées à faire des tâches très concrètes comme "faire du lavage [...] apporter une brosse à dents" (Charlotte), ainsi que des tâches thérapeutiques, étant parfois celles «qui peuvent donner un soutien en santé mentale» (Ophélie).

Parler ici de tâches thérapeutiques soulève une dissemblance entre ce que pensent les différentes participantes. En effet, les opinions divergent quand il est question de savoir si elles s'identifient comme des thérapeutes ou non. À cet effet, Chloé distingue la " thérapie », qui s'appuie sur des protocoles, de " l'intervention », qui n'est pistée que par des approches, des techniques, des valeurs, sans être basée sur des protocoles. Pour elle, les travailleuses sociales ne sont pas des thérapeutes, mais bien des intervenantes. Cela va dans le même sens que ce que souligne Simone, en disant que le travail social « c'est du mou, ce n'est pas très concret, c'est beaucoup des intuitions, des feelings ". II n'y aurait donc pas cet aspect protocolaire dans l'intervention en travail social en général. C'est aussi ce qu'exprime Mia lorsqu'elle dit qu'en travail social, « on n'a pas de tests. On a des approches, mais on n'a pas de tests, d'évaluation. On a des questionnaires, mais ce n'est pas très développé. On n'a pas de matériel qui nous assoit ». 
Mais Mia en vient tout de même à affirmer qu'elle est « une thérapeute au même titre que la psy », simplement qu'elle « travaille les interrelations que la personne a avec son environnement. Elle [la psy] travaille le soi ».

En plus de ces tâches concrètes et thérapeutiques, les participantes se font solliciter pour une panoplie d'autres tâches. En fait, Zoé explique que "quand on ne sait pas trop c'est qui qui devrait faire ça, c'est souvent moi qui le fait. C'est pour ça que, des fois, j'ai l'impression un peu de ramasser les choses qui sont laissées entre deux chaises » (Zoé). Elle se retrouve donc avec une variété si grande de tâches que, parfois, elle aurait « le goût de répondre au téléphone et de dire "département des miracles, bonjour" ». En réalité, ce goût de parler de miracles provient non seulement de la variété des tâches qui sont demandées aux travailleuses sociales, mais aussi parfois de la complexité de celles-ci. Dans ce sens, il semble que les travailleuses sociales sont surtout sollicitées pour intervenir dans les « situations qui sont complexes [...] les dossiers nid de guêpes » (Anaïs).

Prendre en charge cette complexité de tâches et de situations sociales vient grandement de leur rôle de «facilitateurs » (Anaïs) du processus de réadaptation, tant pour la clientèle que pour les collègues. C'est ce qui fait dire à Anaïs que "souvent, notre intervention, ça leur permet de faciliter la leur », et ce, de plusieurs façons. D'abord en travaillant à la cohésion des services offerts. Ce rôle leur est laissé en raison de la vision globale qu'elles posent sur la situation de la clientèle.

C'est comme si, les membres de l'équipe, ensemble, on représentait chacun des carrés et qu'on met les carrés un à côté de l'autre, mais pour que ça prenne sens pour le patient et sa famille, moi, mon rôle, c'est comme de... pas d'arrondir les coins, mais de s'arranger pour que tout ça glisse. Pour que, finalement, ça soit « circulable » un peu. Mettre comme du sens autour de ça pour que ça roule bien et que ça prenne forme pour lui. (Zoé)

Pour que tout roule, elles sont appelées à faire des tâches de toutes sortes. Cela va même parfois jusqu'à la prise en charge de rôles qui reviendraient normalement à un autre membre de l'équipe, par exemple en compensant les pénuries de personnel. Elles peuvent ainsi devenir le «psychologue du pauvre » (Ophélie) dans une équipe où il n'y a pas de psychologue.

Elles rapportent aussi jouer un rôle auprès de l'équipe, en tant que « protecteur de la famille [...] porte-parole de la famille » (Simone). Elles aident alors à redonner du pouvoir à la clientèle, parfois même pour refuser des recommandations en provenance de l'équipe. En même temps, bien que « les recommandations aux proches viennent des autres membres de l'équipe, [...] c'est moi qui vais en faire le suivi avec le proche »(Romane). Elles sont donc dans un rôle de porteparole de l'équipe auprès des proches, et de porte-parole des proches auprès de l'équipe. Cela les place dans la position idéale pour contribuer à apaiser les tensions qui se créent parfois entre l'équipe et la famille. C'est peut-être pourquoi « souvent, c'est l'équipe, à un moment donné qui commence la réadaptation, ils voient, wow ! Ça va être compliqué et, là, ils [me] font une référence »(Chloé). Elles jouent donc divers rôles pour favoriser le partenariat entre l'équipe, l'usager et ses proches.

Les conceptions du travail social qu'ont les participantes se concrétisent donc, dans le milieu de la réadaptation en déficience physique, par de nombreux rôles qu'elles jouent auprès des usagers 
et leurs proches. C'est ce qui nous amène à affirmer qu'elles font de tout, même des miracles, pour faciliter le travail de réadaptation de leurs collègues.

\section{Nos résultats mis en rapport avec les écrits scientifiques}

Au début du présent article, nous avons défini le travail social, en cohérence avec l'approche écologique, par l'importance qu'il accorde au lien entre l'individu et son environnement (Bronfenbrenner, 1994). Alors qu'une survalorisation des facteurs individuels est déplorée en réadaptation en déficience physique (Fougeyrollas, 2010), les travailleurs sociaux sont vus comme portant une vision disciplinaire pouvant faire une plus grande place aux facteurs environnementaux, jusqu'ici peu mis de l'avant. À travers les résultats présentés, il est possible de voir que les travailleuses sociales sont effectivement porteuses de cette vision globale, centrée sur le lien entre l'individu et son environnement. Par contre, déjà au niveau conceptuel, une tendance à l'individualisation se remarque. En effet, la place laissée par certaines à ce qu'elles appellent le « clinique », avec tout l'aspect psychologisant que cela comporte, les éloigne de cette jonction entre une personne et son environnement, survalorisant encore les facteurs individuels.

De plus, par la définition que les participantes sous-entendent au terme « environnement », un autre pas est fait vers l'individualisation. En effet, alors que l'approche écologique propose d'y inclure l'ensemble des facteurs environnementaux, les participantes ont une vision moins large de cet environnement. Diverses hypothèses peuvent être émises pour expliquer cela et mériteraient d'être explorées davantage. Par exemple, l'accessibilité aux membres de l'entourage moins proches de l'usager est souvent plus difficile. Aussi, les interventions sur les réalités sociopolitiques et socioculturelles ont la réputation de donner moins de résultats à court terme, pouvant expliquer le choix de se centrer sur des interventions qui soulageront une souffrance réelle de façon plus immédiate. Aussi, il ne faut pas oublier que, bien que leurs interventions portent sur des sphères spécifiques de l'environnement, il est possible que les autres sphères soient aussi prises en considération ou qu'elles soient, à l'occasion, ciblées par les participantes. II demeure tout de même que cette définition du concept d'environnement les positionne en divergence avec les conceptions que propose l'approche écologique.

Un autre aspect des données qui mérite notre attention est l'influence du contexte de travail sur la façon dont se concrétisent les conceptions du travail social des participantes. À ce sujet, plusieurs auteurs s'entendent pour dire que l'identité professionnelle se forme à travers un processus de socialisation et d'interactions sociales, impliquant l'Autre (Chouinard et Couturier, 2006). Par contre, plusieurs laissent entendre que les contextes organisationnels se positionnent en porte-à-faux avec certaines caractéristiques du travail social, particulièrement avec ses valeurs, ce qui ne permet alors pas le développement ou le maintien de pratiques éthiques (Pullen-Sansfacon, 2011). II est alors recommandé aux travailleurs sociaux de ne pas laisser le contexte trop influencer leur construction identitaire. Cette tension entre le désir de se définir soimême et celui de s'adapter à son contexte de travail est aussi présente chez les participantes. Toutefois, ce ne sont pas les mêmes participantes qui tiennent un discours et l'autre. En effet, alors que certaines disent qu'il est normal et acceptable de s'adapter à son contexte de travail, d'autres vont plutôt soutenir qu'il ne faut pas se laisser définir par lui, mais plutôt se définir soimême pour ensuite affirmer - imposer - cette définition en contexte. 
En ce qui concerne les rôles que les participantes disent jouer, plusieurs cohérences avec les écrits sont notables. Tout d'abord, cette idée qu'elles ont un rôle de généraliste en est une qui est identifiée par plusieurs (Prud'homme, 2011; Pullen-Sansfaçon et Cowden, 2012). De plus, comme les participantes le soulignent aussi, plusieurs auteurs affirment que ce rôle de généralistes rend difficile l'identification de ce que font les travailleuses sociales. À ce sujet, VatzLaaroussi (RUFUTS, 1998), propose de «parler au pluriel des identités professionnelles » ( $p$. 52), puisque l'identité professionnelle en travail social couvre si large que d'en chercher le dénominateur commun signifie soit ne rien trouver, soit trouver quelque chose qui ne présente qu'un intérêt limité.

Finalement, il faut revenir à la question posée en début d'article à savoir si les travailleurs sociaux en réadaptation physique se définissent dans un rôle supplétif, de deuxième plan. En réponse à cette question, il apparaît que plusieurs aspects de leurs rôles peuvent être considérés comme supplétifs. Par exemple, combler la pénurie de personnel en assumant un rôle normalement joué par un autre intervenant est un rôle de suppléance, supplétif. Aussi, leur rôle de facilitateur les positionne en soutien à l'équipe, ce qui peut être perçu comme un rôle supplétif, en supplément aux interventions de réadaptation de leurs collègues. Cela dit, même s'il apparaît que les participantes aient un rôle pouvant être considéré supplétif, ce dernier n'est en rien secondaire. En effet, lorsqu'elles sont sollicitées pour les diverses tâches qu'on attend d'elles, c'est parce que ces tâches sont considérées comme nécessaires au bon déroulement de la réadaptation. Aussi, si on dit qu'elles facilitent la réadaptation que font leurs collègues, c'est que, sans elles, cette réadaptation serait plus ardue, peut-être même compromise. C'est donc dans une posture de généraliste ayant un rôle supplétif qu'elles font leur place, une place essentielle à la réadaptation.

\section{Conclure pour mieux continuer}

Grâce à des entrevues individuelles avec des travailleuses sociales intervenant en réadaptation en déficience physique depuis un minimum d'un an, nous avons pu explorer la façon dont s'exprime leur identité professionnelle dans ce contexte de travail. Cela a permis de voir que les participantes conçoivent le travail social d'une façon présentant cohérences et divergences avec l'approche écologique (Bronfenbrenner, 1994), approche utilisée en travail social et en réadaptation physique - à travers le $\mathrm{PPH}$. Nous avons aussi vu que l'actualisation de leurs conceptions est teintée par le contexte de travail, à la fois par l'interprétation du mandat auquel les participantes doivent se plier que par la négociation des zones grises qu'elles partagent avec des collègues. Tout cela se concrétise ensuite par un rôle de généraliste, faisant une grande diversité de tâches, principalement dans l'optique de faciliter le travail des autres membres de l'équipe. Cela les positionne dans un rôle qui nous apparaît supplétif, mais tout de même essentiel au bon déroulement du processus de réadaptation.

Cela dit, est-ce que cela les positionne de façon à pouvoir augmenter l'attention mise sur les facteurs environnementaux, jusqu'à maintenant négligés, plutôt que sur les facteurs individuels dans la compréhension de la déficience physique et des services de réadaptation ? À cette question, nous répondons qu'elles interviennent dans ce sens, mais que leur position souvent perçue comme de second ordre ne leur donne pas toujours le pouvoir de faire la différence. Il est tout de même encourageant de savoir qu'elles ne sont pas seules à viser à laisser une plus grande place aux facteurs environnementaux, et qu'elles y travaillent réellement. II faut tout 
simplement garder en tête que, elles aussi, comme les personnes ayant une déficience physique, doivent gérer des contraintes environnementales qui limitent et teintent les possibles qui s'offrent à elles.

\section{CRÊTE, Josianne}

\section{RÉFÉRENCES}

ASSSGIM (Agence de la santé et des services sociaux de la Gaspésie-liles-de-la-Madeleine) (2006). Plan d'organisation des services de réadaptation fonctionnelle intensive pour la région de la Gaspésie et des îles-de-la-Madeleine. 62 p.

Blewett, J., J. Lewis, et J. Tunstill (2007). The Changing Roles and Tasks of Social Work: A Literature Informed Discussion Paper, General Social Care Council, Londres.

Bouchard, C. (1987). «Intervenir à partir de l'approche écologique : au centre, l'intervenante ». Service social, vol. 36, $\mathrm{n}^{\text {os }}$ 2-3, p. 454-477.

Bouquet, B. (2003). Éthique et travail social. Une recherche de sens, Paris, Dunod.

Bronfenbrenner, U. (1994). « Ecological model of human development ». Dans T. Husén et T. N. Postlethwaite (dir.), International Encyclopedia of Education ( $2^{\mathrm{e}}$ éd., vol. 3), Oxford, Elsevier, p. 1643-1647.

Charmaz, K. (2000). « Grounded theory: Objectivist and constructivist methods ». Dans N. K. Denzin et Y. S. Lincoln, Handbook of Qualitative Research ( $2^{\mathrm{e}}$ éd.), Thousand Oaks (CA), Sage, p. 509-535.

Chouinard, I., et Y. Couturier (2006). « Identité professionnelle et souci de soi en travail social ». Nouvelles pratiques sociales, vol. 19, $\mathrm{n}^{\circ} 1$, p. 176-182.

Dubar, C. (2000). La socialisation. Construction des identités sociales et professionnelles (3éd. rev.), Paris, Armand Colin, 255 p.

Duchesne, C., et L. Savoie-Zajc (2005). «L'engagement professionnel d'enseignantes du primaire : une démarche inductive de théorisation ». Recherches qualitatives, vol. $25, \mathrm{n}^{\circ} 2$, p. 69-95.

Fougeyrollas, P. (2010). La funambule, le fil et la toile. Transformations réciproques du sens du handicap. Québec, Presses de l'Université Laval, coll. « Sociétés, cultures et santé ».

Fougeyrollas, P., R. Cloutier, H. Bergeron, J. Côté et G. St-Michel (1998). Classification québécoise : Processus de production du handicap, Québec, Réseau international sur le Processus de production du handicap, $166 \mathrm{p}$. 
Granja, B. (2008). "Éléments de construction identitaire professionnelle des assistants de service social en formation ». Travail-Emploi-Formation, n 8, p. 21-38.

Guillemette, F., et J. R. Lapointe (2012). « Illustration d'un effort pour demeurer fidèle à la spécificité de la méthodologie de la théorisation enracinée (Grounded Theory) 》. Dans J. Luckerhoff et F. Guillemette (dir.), Méthodologie de la théorisation enracinée. Fondements, procédures et usages, Québec, Presses de l'Université du Québec, p. 11-35.

INESSS (Institut national d'excellence en santé et en services sociaux) (2012a). L'organisation et la prestation de services de réadaptation pour les personnes ayant subi un accident vasculaire cérébral (AVC) et leurs proches. ETMIS, vol. 8, nº 9.

INESSS (Institut national d'excellence en santé et en services sociaux) (2012b). Les caractéristiques, l'historique et l'implantation du continuum de services en traumatologie du Québec (1991-2012). Montréal, INESSS, 85 p.

Legault, G. A. (2008). L'identité : rôles, composantes et fonctions relationnelles. Résumé de présentation faite au Colloque de l'APEC.

OTSTCFQ (Ordre des travailleurs sociaux et des thérapeutes conjugaux et familiaux du Québec) (2012). Référentiel des compétences des travailleuses sociales et des travailleurs sociaux. OTSTCFQ.

Payne, M. (2004). « Social Work Practice Identities: An Agency Study of a Hospice ». Practice: Social Work in Action, vol. 16, n 1, p. 5-15.

Prud'homme, J. (2011). Professions à part entière. Histoire des ergothérapeutes, orthophonistes, physiothérapeutes, psychologues et travailleuses sociales au Québec. Montréal, Presses de I'Université de Montréal, $223 \mathrm{p}$.

Pullen-Sansfaçon, A. (2011). «Ethics and Conduct in Self-directed Groupwork: Some Lessons for the Development of a More Ethical Social Work Practice ». Ethics and Social Welfare, vol. $5, n^{\circ} 4$, p. 361-379.

Pullen-Sansfaçon, A., et S. Cowden (2012). The Ethical foundations of social work. Harlow (England), Pearson.

RUFUTS (Regroupement des Unités de formation universitaire en Travail social) (1998). L'avenir de la profession du travail social : point de vue des universitaires. Compte rendu d'une Journée de réflexion, Montréal. Récupéré le 2013-05-31 de <http://www.unites.uqam.ca/rufuts/Archives1998.html>.

Ruohotie-Lyhty, M. (2013). "Struggling for a professional identity: Two newly qualified language teachers' identity narratives during the first years at work ». Teaching and Teacher Education, vol. $30, n^{\circ} 1$, p. 120-129. 
Strauss, A. (1992). La trame de la négociation. Sociologie qualitative et interactionnisme. Paris, L'Harmattan, $319 \mathrm{p}$. 